\title{
Algebraic characterization of finite (branched) coverings
}

by

\author{
M. A. Mulero (Badajoz)
}

\begin{abstract}
Every continuous map $X \rightarrow S$ defines, by composition, a homomorphism between the corresponding algebras of real-valued continuous functions $C(S) \rightarrow C(X)$. This paper deals with algebraic properties of the homomorphism $C(S) \rightarrow C(X)$ in relation to topological properties of the map $X \rightarrow S$. The main result of the paper states that a continuous map $X \rightarrow S$ between topological manifolds is a finite (branched) covering, i.e., an open and closed map whose fibres are finite, if and only if the induced homomorphism $C(S) \rightarrow C(X)$ is integral and flat.
\end{abstract}

Introduction. The aim of this paper is to characterize finite coverings $X \rightarrow S$ between topological spaces by means of the algebraic properties of the induced homomorphism $C(S) \rightarrow C(X)$ between the algebras of realvalued continuous functions.

Our starting point is the well-known result which states that, in the realm of realcompact spaces, every space $X$ is determined by the algebra $C(X)$ of all real-valued continuous functions defined on it, and that continuous maps between such spaces are in one-to-one correspondence with homomorphisms between their algebras of continuous functions. In view of this equivalence, it seems natural to expect that every property concerning topological spaces and continuous maps may be characterized in terms of the algebras of continuous functions.

By a finite (branched) covering we mean an open and closed continuous map $\pi: X \rightarrow S$ with finite fibres. Note that a finite covering is not necessarily a local homeomorphism (for example, $\pi: \mathbb{C} \rightarrow \mathbb{C}, \pi(z)=z^{2}$ ). The set of points in $X$ at which $\pi$ is not a local homeomorphism is called the branch set of $\pi$. In [15] the author gives an algebraic characterization of this branch set in terms of finite $C(S)$-subalgebras $A$ of $C(X)$ that separate points in $X$ and the module $\Omega_{A / C(S)}$ of its Kähler differentials.

1991 Mathematics Subject Classification: 54C10, 54C40, 13C11.

Key words and phrases: branched covering, open and closed map, ring of continuous functions, flat homomorphism, integral homomorphism. 
The main result of the present paper is the following characterization of finite coverings between topological manifolds:

Theorem. Let $X$ and $S$ be topological manifolds. A continuous map $X \rightarrow S$ is a finite covering if and only if the induced homomorphism $C(S) \rightarrow$ $C(X)$ is integral and flat. A continuous map $X \rightarrow S$ is an unbranched finite covering if and only if the induced homomorphism $C(S) \rightarrow C(X)$ is finite and flat.

The main difficulty is to show that $C(S) \rightarrow C(X)$ is flat. This difficulty was to be expected, since the flatness is a considerably technical algebraic property and we must obtain it starting from topological properties.

The above formulation is inspired by similar results in algebraic geometry, mainly by Zariski's Main Theorem that relates the finiteness of the fibres of a morphism between algebraic varieties to the finiteness of the corresponding morphism between rings of algebraic functions ([4, p. 240] or [8, Theorem 8.12.6]).

Differential geometry is another geometric context where the general principle of mutual determination between spaces (differentiable manifolds) and algebras of $\left(C^{\infty}\right)$ functions remains valid. We believe that similar results might be stated for $C^{\infty}$ mappings between differentiable manifolds by means of Malgrange's Preparation Theorem ([11, Theorem 4.1] or [16, pp. 187$190])$, but we shall not treat this case here.

1. Preliminaries. For rings of continuous functions, we use the same terminology and notation as in [6]. For algebraic concepts, the reader may consult [1], [3] or [13]. Throughout the paper we work with the prime spectrum of $C(X)$, so that we now review some relevant definitions and results.

The set of prime ideals in a ring $A$, i.e., the prime spectrum, is denoted by $\operatorname{Spec} A$. We consider it to be endowed with the Zariski topology: For any subset $C$ of $A$, let $\mathrm{V}(C)=\{\mathfrak{p} \in \operatorname{Spec} A: C \subseteq \mathfrak{p}\}$ and take as closed sets in Spec $A$ all subsets of the form $\mathrm{V}(C)$. If $f \in A$, we put $\mathrm{D}(f)=\operatorname{Spec} A-\mathrm{V}(f)$. The collection of those open sets forms a basis of the topology of Spec $A$.

Each ring homomorphism $h: A \rightarrow B$ induces a continuous map $h^{*}$ : Spec $B \rightarrow$ Spec $A$ which sends $\mathfrak{p} \in \operatorname{Spec} B$ to $h^{-1}(\mathfrak{p}) \in \operatorname{Spec} A$.

If $h: A \rightarrow B$ is a surjective homomorphism, then $h^{*}$ is a homeomorphism between $\operatorname{Spec} B$ and the closed subset $\mathrm{V}(\operatorname{ker}(h))$ of $\operatorname{Spec} A$.

If $B$ is the ring of fractions of $A$ with respect to a multiplicatively closed subset $S$ of $A$, i.e., $B=\{a / s: a \in A, s \in S\}$ and $h: A \rightarrow B$ is the natural homomorphism, $h(a)=a / 1$, then $h^{*}$ is a homeomorphism between $\operatorname{Spec} B$ and the subset $\{\mathfrak{p} \in \operatorname{Spec} A: \mathfrak{p} \cap S=\emptyset\}$ of $\operatorname{Spec} A$.

Let $M$ be an $A$-module. For $a \in A$, we denote by $M_{a}$ the $A$-module of fractions with respect to the multiplicatively closed subset $\left\{a^{n}: n \in \mathbb{N}\right\}$. 
If $\mathfrak{p}$ is a prime ideal in $A$, we denote by $M_{\mathfrak{p}}$ the localization, or module of fractions, of $M$ with respect to the multiplicatively closed subset $A-\mathfrak{p}$.

Throughout the paper, $C(X)$ is the algebra of all real-valued continuous functions on $X$ and $C^{*}(X)$ the subalgebra of bounded functions. Every $f \in C(X)$ can be written as the fraction $f=f \cdot\left(1+f^{2}\right)^{-1} /\left(1+f^{2}\right)^{-1}$, so that $C(X)$ is the ring of fractions of $C^{*}(X)$ with respect to the multiplicatively closed subset $M_{X}=\left\{f \in C^{*}(X): 0 \notin f(X)\right\}$. Hence, $\operatorname{Spec} C(X)$ is naturally homeomorphic to the subspace $\left\{\mathfrak{p} \in \operatorname{Spec} C^{*}(X): \mathfrak{p} \cap M_{X}=\emptyset\right\}$ of $\operatorname{Spec} C^{*}(X)$.

The zero-set of a function $f \in C(X)$ is $\mathrm{Z}(f)=\{x \in X: f(x)=0\}$, and $\operatorname{coz}(f)=X-\mathrm{Z}(f)$ is the cozero-set of $f$.

An ideal $I$ of $C(X)$ is said to be a z-ideal if $\mathrm{Z}(f) \subseteq \mathrm{Z}(g)$ and $f \in I$ imply $g \in I$.

If $U$ is a cozero-set, $U=\operatorname{coz}(f)$, then $C(U)$ is the localization, or ring of fractions, of $C(X)$ with respect to the multiplicatively closed subset $S_{U}=$ $\{h \in C(X): 0 \notin h(U)\}$, i.e., $C(U)=\{f / h: f, h \in C(X), 0 \notin h(U)\}$ (see $[2])$.

For the bounded case we have the following lemma.

Lemma 1.1. Let $f \in C^{*}(X)$. If $U=\operatorname{coz}(f)$ then $C^{*}(X)_{f}=C^{*}(U)_{f}$.

P r o of. If $g \in C^{*}(U)$, it is clear that the function $g^{\prime}$ defined by $g^{\prime}=g \cdot f$ on $U=\operatorname{coz}(f)$ and $g^{\prime}=0$ on $\mathrm{Z}(f)$ is continuous on $X$, and in the ring of fractions $C^{*}(U)_{f}$ we can write $g / 1=g^{\prime} / f$.

It is well known that the maximal spectrum of $C(X)$, i.e., the subspace of Spec $C(X)$ consisting of all maximal ideals in $C(X)$, is a Hausdorff compact space. Every completely regular space $X$ may be identified with a dense subspace of the maximal spectrum of $C(X)$ : each point $x \in X$ defines the maximal ideal $\mathfrak{m}_{x}=\{f \in C(X): f(x)=0\}$, and the maximal spectrum of $C(X)$ can be identified with $\beta X$, the Stone-Cech compactification of $X$. The same holds for the maximal spectrum of $C^{*}(X)$.

If $M$ is a $C(X)$-module, we write $M_{x}$ instead of $M_{\mathfrak{m}_{x}}$.

Unless otherwise specified, $X$ and $S$ will be completely regular spaces.

Every map $\pi: X \rightarrow S$ defines, by composition, two homomorphisms of rings $C(S) \rightarrow C(X)$ and $C^{*}(S) \rightarrow C^{*}(X)$. Given $g \in C(S)$, if no confusion is possible, the function $g \circ \pi$ will also be denoted by $g$. The maps between spectra induced by these homomorphisms of rings are denoted by $\pi_{s}: \operatorname{Spec} C(X) \rightarrow \operatorname{Spec} C(S)$ and $\pi^{*}: \operatorname{Spec} C^{*}(X) \rightarrow \operatorname{Spec} C^{*}(S)$, respectively. The extension of $\pi$ to the Stone-Cech compactification is denoted by $\pi_{\beta}: \beta X \rightarrow \beta S$. 


\section{Finite coverings}

Definitions 2.1. Let $X$ and $S$ be two arbitrary topological spaces (not necessarily completely regular, nor even Hausdorff). A map $\pi: X \rightarrow S$ is separated if any two points in the same fibre have disjoint neighbourhoods in $X$. The map $\pi$ is said to be finite if it is proper (closed and with compact fibres), separated and each fibre is a finite set.

Definitions 2.2. Let $X$ and $S$ be arbitrary topological spaces. A map $\pi: X \rightarrow S$ is a trivial covering if the space $X$ has a cover by pairwise disjoint open sets, each homeomorphic to $S$ via $\pi$.

A map $\pi: X \rightarrow S$ is an unbranched covering if every point of $S$ has a neighbourhood $U$ such that $\pi: \pi^{-1}(U) \rightarrow U$ is a trivial covering.

By a finite covering we mean a finite and open map $\pi: X \rightarrow S$. Since the image of a finite covering is an open and closed subset of $S$, there is no loss of generality for our purposes in assuming that every finite covering is a surjective map.

Definition 2.3. Let $\pi: X \rightarrow S$ be a finite covering between arbitrary topological spaces. Let $s$ be a point in $S$ and $\pi^{-1}(s)=\left\{x_{1}, \ldots, x_{n}\right\}$. We say that a neighbourhood $U$ of $s$ is special for $\pi$ if

$$
\pi^{-1}(U)=U_{1} \sqcup \ldots \sqcup U_{n},
$$

where $U_{i}$ is a neighbourhood of $x_{i}$ such that

$$
\pi\left(U_{i}\right)=U \quad \text { for every } i, \quad U_{i} \cap U_{j}=\emptyset \quad \text { if } i \neq j .
$$

It is not difficult to prove that each $s \in S$ has a basis of special neighbourhoods.

Definitions 2.4. The branch set of a finite covering $\pi: X \rightarrow S$ is the set of all points in $X$ where $\pi$ is not a local homeomorphism. We denote this closed set by $B_{\pi}$, or simply $B$ if no confusion is possible.

The closed set $\pi\left(B_{\pi}\right)$ is denoted by $R_{\pi}$, or simply $R$, and its complement $S-R$ by $S_{0}$. Clearly, a point $s \in S$ belongs to $S_{0}$ if and only if the cardinality of the fibres of $\pi$ is constant on some neighbourhood of $s$.

If $X_{0}=\pi^{-1}\left(S_{0}\right)$ then the restriction $\pi: X_{0} \rightarrow S_{0}$ is an unbranched covering and is said to be the unbranched part of $\pi$.

EXAMPLES 2.5. The study of unbranched coverings is a classical subject. (It is well known that unbranched coverings are closely connected with the study of the fundamental group, see for instance [12].) The following examples show that the class of finite branched coverings includes some interesting kinds of maps.

It is a classical result that non-constant analytic maps between Riemann surfaces are open, hence any non-constant analytic map with finite fibres between compact Riemann surfaces is a finite covering. 
It is well known and easy to prove that if a group $G$ acts on a space $X$ (i.e., there is a morphism of groups from $G$ to the group of homeomorphisms of $X$ ) then the natural projection map of $X$ onto its quotient space $\pi: X \rightarrow$ $X / G$ is an open map. If $X$ is Hausdorff and $G$ is finite, this map is a finite covering.

In order to study branched coverings from the algebraic point of view, we must define the concepts of "degree" of a finite covering, and "multiplicity" or "index of branching" at a branching point.

Definitions 2.6. Let $\pi: X \rightarrow S$ be a finite covering with unbranched part $\pi: X_{0} \rightarrow S_{0}$. Given a point $x \in X$, we say that a number $b(x) \in \mathbb{N}$ is the index of branching of $\pi$ at $x$ if there exists a basis of neighbourhoods $U$ of $x$ such that $U \cap X_{0} \neq \emptyset$ and the cardinality of the fibres of the unbranched covering $\pi: U \cap X_{0} \rightarrow \pi\left(U \cap X_{0}\right)$ is $b(x)$. It is easy to prove that the index of branching at $x$, if it exists, is unique.

If the index of branching of $\pi: X \rightarrow S$ exists at every point in $X$, we define the degree of $\pi$ at $s \in S$ as the number $\sum_{x \in \pi^{-1}(s)} b(x)$. It is easy to prove that the degree is locally constant, so that there is no loss of generality if we assume from now on that it is constant.

By a covering of degree $n$ we mean a finite covering $\pi: X \rightarrow S$ such that the index of branching exists at every point in $X$ and the degree of $\pi$ at every point in $S$ is $n$.

Note that if $\pi: X \rightarrow S$ is a covering of degree $n$ then the cardinality of each fibre of the unbranched part $\pi: X_{0} \rightarrow S_{0}$ is $n$.

EXAMPLES 2.7. It is clear that every unbranched covering has finite degree.

If a finite covering $\pi: X \rightarrow S$ of a locally connected space $S$ is such that the closed set $R$ is thin, i.e., it has an empty interior and each $s \in S$ has a basis of connected neighbourhoods $U$ such that $U \cap S_{0}$ is connected, then the index of branching of $\pi$ exists at every point in $X$ and therefore the covering has finite degree. Explicitly: if $s \in S, \pi^{-1}(s)=\left\{x_{1}, \ldots, x_{n}\right\}$ and $U$ is a special neighbourhood of $s$ such that $U \cap S_{0}$ is connected and $\pi^{-1}(U)=U_{1} \sqcup \ldots \sqcup U_{n}$, where $x_{i} \in U_{i}$ and $\pi\left(U_{i}\right)=U$, then all fibres of the unbranched covering $\pi: U_{i} \cap X_{0} \rightarrow U \cap S_{0}$ have the same cardinality $b\left(x_{i}\right)$.

In [10] it is proved that, if $X$ and $S$ are topological manifolds, then the supremum of the cardinalities of the fibres of any finite covering $\pi: X \rightarrow S$ is finite and the closed set $R$ is thin, so that every finite covering between topological manifolds has finite degree.

Recall that a ring homomorphism $h: A \rightarrow B$ is said to be integral if every element of $B$ is a root of a monic polynomial in $A[x]$. 
Proposition 2.8. If $\pi: X \rightarrow S$ is a covering of finite degree then the induced homomorphisms $C(S) \rightarrow C(X)$ and $C^{*}(S) \rightarrow C^{*}(X)$ are integral.

Proof. Let $n$ be the degree of $\pi$. If $\pi$ is an unbranched covering, it is clear that for every $f \in C(X)$ the functions $S_{k}(f)$ defined for $s \in S$ by

$$
S_{k}(f)(s)=\sum_{1 \leq i_{1}<\ldots<i_{k} \leq m} f\left(x_{i_{1}}\right) \ldots f\left(x_{i_{k}}\right),
$$

where $\left\{x_{1}, \ldots, x_{m}\right\}=\pi^{-1}(s)$, are continuous on $S$. Obviously $f$ is a root of the polynomial

$$
t^{n}-S_{1}(f) t^{n-1}+\ldots+(-1)^{n} S_{n}(f) .
$$

In the branched case, $f$ will be a root of the same polynomial provided we define the value of $(-1)^{k} S_{k}(f)$ at $s$ to be the coefficient of $t^{n-k}$ in the polynomial

$$
\left(t-f\left(x_{1}\right)\right)^{b\left(x_{1}\right)} \ldots\left(t-f\left(x_{m}\right)\right)^{b\left(x_{m}\right)}, \quad\left\{x_{1}, \ldots, x_{m}\right\}=\pi^{-1}(s) .
$$

It is easy to prove that these functions $S_{k}(f)$ are continuous and that $S_{k}(f) \in C^{*}(S)$ whenever $f \in C^{*}(X)$.

3. Extensions of a finite covering. This section is devoted to studying the extensions $\pi^{*}: \operatorname{Spec} C^{*}(X) \rightarrow \operatorname{Spec} C^{*}(S), \pi_{s}: \operatorname{Spec} C(X) \rightarrow \operatorname{Spec} C(S)$ and $\pi_{\beta}: \beta X \rightarrow \beta S$ of a finite covering $\pi: X \rightarrow S$.

To prove that the homomorphism $C(S) \rightarrow C(X)$ induced by a covering $\pi: X \rightarrow S$ of finite degree is flat, we use the fact that these extensions are also coverings of finite degree. To prove this, we start from the following results for open and proper maps obtained by the author in [14].

Theorem 3.1. If $\pi: X \rightarrow S$ is open and proper then:

(i) The extensions $\pi^{*}: \operatorname{Spec} C^{*}(X) \rightarrow \operatorname{Spec} C^{*}(S)$ and $\pi_{\beta}: \beta X \rightarrow \beta S$ are also open and proper.

(ii) $C(X)$ is the ring of fractions of $C^{*}(X)$ with respect to the multiplicatively closed subset $M_{S}=\left\{g \in C^{*}(S): 0 \notin g(S)\right\}$.

(iii) $\pi^{*}: \operatorname{Spec} C^{*}(X) \rightarrow \operatorname{Spec} C^{*}(S)$ transforms $\operatorname{Spec} C^{*}(X)-\operatorname{Spec} C(X)$ into $\operatorname{Spec} C^{*}(S)-\operatorname{Spec} C(S)$.

(iv) $\pi_{s}: \operatorname{Spec} C(X) \rightarrow \operatorname{Spec} C(S)$ is open and proper.

Proposition 3.2. If $\pi: X \rightarrow S$ is a covering of degree $n$ then its extensions $\pi^{*}: \operatorname{Spec} C^{*}(X) \rightarrow \operatorname{Spec} C^{*}(S), \pi_{s}: \operatorname{Spec} C(X) \rightarrow \operatorname{Spec} C(S)$ and $\pi_{\beta}: \beta X \rightarrow \beta S$ are coverings of the same degree $n$.

Proof. The map $\pi_{\beta}: \beta X \rightarrow \beta S$ is of course proper, and it is known that it is open (see [9] or [14, Corollary 1.7]). The fibre of any point in $\beta X$ has no more than $n$ points, because if the fibre over $p \in \beta S$ has more than $n$ points, then so does the fibre over every point in a neighbourhood of $p$. This, 
however, cannot occur, because $S$ is dense in $\beta S$ and, since $\pi$ is proper, one has $\pi_{\beta}^{-1}(S)=X[5$, Theorem 3.7.16].

By Theorem 3.1, $\pi_{s}$ and $\pi^{*}$ are open and proper. Since the induced homomorphism $C(S) \rightarrow C(X)$ is integral (2.8), there is no inclusion between prime ideals belonging to the same fibre $[1$, Corollary 5.9]. This implies that $\pi_{s}$ is a separated map. Explicitly: Let $\mathfrak{q}_{1}$ and $\mathfrak{q}_{2}$ be two prime ideals in $C(X)$ such that $\pi_{s}\left(\mathfrak{q}_{1}\right)=\pi_{s}\left(\mathfrak{q}_{2}\right)$. Since there is no inclusion between $\mathfrak{q}_{1}$ and $\mathfrak{q}_{2}$ and the prime ideals in $C(X)$ containing a given prime ideal form a chain [6, $14.3(\mathrm{c})]$, there are no prime ideals in $C(X)$ contained in both $\mathfrak{q}_{1}$ and $\mathfrak{q}_{2}$. The prime ideals contained simultaneously in $\mathfrak{q}_{1}$ and $\mathfrak{q}_{2}$ are just the prime ideals in the ring $\left(C(X)_{\mathfrak{q}_{1}}\right)_{\mathfrak{q}_{2}}$, so that $\left(C(X)_{\mathfrak{q}_{1}}\right)_{\mathfrak{q}_{2}}=0$, and therefore there are functions $f_{1} \notin \mathfrak{q}_{1}$ and $f_{2} \notin \mathfrak{q}_{2}$ such that $f_{1} \cdot f_{2}=0$. Clearly, $\mathrm{D}\left(f_{1}\right)$ and $\mathrm{D}\left(f_{2}\right)$ are disjoint neighbourhoods of $\mathfrak{q}_{1}$ and $\mathfrak{q}_{2}$ in Spec $C(X)$. Clearly, the same holds for $\pi^{*}$ since the induced homomorphism $C^{*}(S) \rightarrow C^{*}(X)$ is also integral. Now, we may prove that the fibres of $\pi_{s}$ and $\pi^{*}$ have no more than $n$ points with the arguments given above for $\pi_{\beta}$. Thus, for all these maps we only have to prove the existence of the index of branching at every point.

The map $\pi^{*}$ transforms $\operatorname{Spec} C^{*}(X)-\operatorname{Spec} C(X)$ into $\operatorname{Spec} C^{*}(S)-$ Spec $C(S)$ (by 3.1(iii)), i.e., $\left(\pi^{*}\right)^{-1}(\operatorname{Spec} C(S))=\operatorname{Spec} C(X)$. Since $\pi^{*}$ is separated, it maps $\operatorname{Spec} C^{*}(X)-\beta X$ into $\operatorname{Spec} C^{*}(S)-\beta S$, i.e., $\left(\pi^{*}\right)^{-1}(\beta S)$ $=\beta X$. Moreover, $\left(\pi^{*}\right)^{-1}(S)=X$, because $\pi$ is proper, and since $S$ is dense in Spec $C^{*}(S)$, this implies that the unbranched part of $\pi^{*}$ is just the restriction of $\pi^{*}$ to the subspace of $\operatorname{Spec} C(X)$ consisting of the fibres that have exactly $n$ points. Clearly, the same holds for $\pi_{s}$ and $\pi_{\beta}$. Hence, it suffices to prove the existence of the index of branching of $\pi^{*}$ at every point in $\operatorname{Spec} C^{*}(X)$. We do this by induction on $n$.

For $n=1$ it is trivially true. For a covering of degree $n$, it is clear that if the fibre over $\mathfrak{p} \in \operatorname{Spec} C^{*}(S)$ has only one point $\mathfrak{q}$ then the index of branching of $\pi^{*}$ at $\mathfrak{q}$ is $n$.

Let $\mathfrak{p} \in \operatorname{Spec} C^{*}(S)$ and $\left(\pi^{*}\right)^{-1}(\mathfrak{p})=\left\{\mathfrak{q}_{1}, \ldots, \mathfrak{q}_{m}\right\}$, where $m \geq 1$. Since $\pi^{*}$ is a finite covering, we can take a basic neighbourhood $\mathrm{D}(g)$ of $\mathfrak{p}$ special for $\pi^{*}$ such that $\left(\pi^{*}\right)^{-1}(\mathrm{D}(g))=\mathrm{D}\left(g_{1}\right) \sqcup \ldots \sqcup \mathrm{D}\left(g_{m}\right)$, where each $\mathrm{D}\left(g_{i}\right)$ is a neighbourhood of $\mathfrak{q}_{i}$ such that $\pi^{*}\left(\mathrm{D}\left(g_{i}\right)\right)=\mathrm{D}(g)$. If $U=\mathrm{D}(g) \cap S=\operatorname{coz}(g)$ and $U_{i}=\mathrm{D}\left(g_{i}\right) \cap X=\operatorname{coz}\left(g_{i}\right)$, then $\pi^{-1}(U)=U_{1} \sqcup \ldots \sqcup U_{m}$, and for each $i=1, \ldots, m$, the map $\pi: U_{i} \rightarrow U$ is a covering of degree less than or equal to $n-(m-1)$. Hence, by the induction hypothesis, the index of branching exists at every point for the covering

$$
\operatorname{Spec} C^{*}\left(\pi^{-1}(U)\right)=\bigsqcup_{i=1, \ldots, m} \operatorname{Spec} C^{*}\left(U_{i}\right) \rightarrow \operatorname{Spec} C^{*}(U) .
$$

Since $C^{*}(S)_{g}=C^{*}(U)_{g}$ (see 1.1), we find that $\operatorname{Spec} C^{*}(S)_{g}=\mathrm{D}(g)$ is an open subset of $\operatorname{Spec} C^{*}(U)$. We conclude that the index of branching of $\pi^{*}$ 
exists at every point $\mathfrak{q}_{i} \in \pi^{-1}(\mathfrak{p})$, by the following commutative diagram:

$$
\begin{aligned}
& \left(\pi^{*}\right)^{-1}(\mathrm{D}(g))=\bigsqcup_{i} \mathrm{D}\left(g_{i}\right) \simeq \bigsqcup_{i} \operatorname{Spec} C^{*}\left(U_{i}\right)_{g_{i}} \hookrightarrow \bigsqcup_{i} \operatorname{Spec} C^{*}\left(U_{i}\right) \\
& \pi^{*} \downarrow \\
& \mathrm{D}(\mathrm{g}) \simeq \operatorname{Spec} C^{*}(U)_{g} \quad \hookrightarrow \quad \operatorname{Spec} C^{*}(U)
\end{aligned}
$$

Corollary 3.3. Let $\pi: X \rightarrow S$ be a finite covering. If the supremum of the cardinalities of the fibres of $\pi$ is a finite number $n$, then the extensions $\pi^{*}: \operatorname{Spec} C^{*}(X) \rightarrow \operatorname{Spec} C^{*}(S), \pi_{s}: \operatorname{Spec} C(X) \rightarrow \operatorname{Spec} C(S)$ and $\pi_{\beta}:$ $\beta X \rightarrow \beta S$ are finite coverings and the supremum of the cardinalities of the fibres of each of them is also $n$.

Proof. Firstly, note that for $\pi_{\beta}$, the assertion is already proved in 3.2. We also know that $\pi_{s}$ and $\pi^{*}$ are open and proper. Thus, we only have to prove that they are separated and have no more than $n$ points in their fibres. We prove this by induction on $n$. For $n=1$, this is trivially true. Assume that it is also true when the supremum of the cardinalities of the fibres is strictly less than $n$. Since $\left(\pi^{*}\right)^{-1}(\operatorname{Spec} C(S))=\operatorname{Spec} C(X)(3.1($ iii) $)$, it is clear that we can replace $X$ and $S$ with $\beta X$ and $\beta S$ and suppose that both are compact spaces.

Let $R$ be the $\pi$-image of the branch set. Since $S$ is a compact space, the restriction homomorphism $C(S) \rightarrow C(R)$ is surjective and its kernel is the ideal $I_{R}$ of all functions vanishing on $R$. Hence, Spec $C(R)$ is naturally homeomorphic to $\mathrm{V}\left(I_{R}\right) \subseteq \operatorname{Spec} C(S)$. Similarly, $C\left(\pi^{-1}(R)\right)$ is a quotient ring of $C(X)$ and Spec $C\left(\pi^{-1}(R)\right)$ is naturally homeomorphic to $\mathrm{V}\left(I_{\pi^{-1}(R)}\right) \subseteq$ Spec $C(X)$. It is not difficult to prove that $I_{R} \cdot C(X)=I_{\pi^{-1}(R)}$ and therefore $\pi_{s}^{-1}\left(\mathrm{~V}\left(I_{R}\right)\right)=\mathrm{V}\left(I_{R} \cdot C(X)\right)=\mathrm{V}\left(I_{\pi^{-1}(R)}\right)$. The cardinality of the fibres of the points belonging to $R$ is strictly smaller than $n$ and hence, by the induction hypothesis, $\pi_{s}: \operatorname{Spec} C\left(\pi^{-1}(R)\right)=\mathrm{V}\left(I_{\pi^{-1}(R)}\right) \rightarrow \operatorname{Spec} C(R)=\mathrm{V}\left(I_{R}\right)$ is a covering whose fibres have less than $n$ points. It follows that the number of points in the fibre of $\pi_{s}: \operatorname{Spec} C(X) \rightarrow \operatorname{Spec} C(S)$ over any point $\mathfrak{p} \in \mathrm{V}\left(I_{R}\right)$ is strictly less than $n$ and any two points in $\pi_{s}^{-1}(\mathfrak{p})$ have disjoint neighbourhoods in Spec $C\left(\pi^{-1}(R)\right)$. Hence, there is no inclusion between prime ideals in $\pi_{s}^{-1}(\mathfrak{p})$ and, as we saw in the proof of 3.2 , this implies that any two points in $\pi_{s}^{-1}(\mathfrak{p})$ have disjoint neighbourhoods in $\operatorname{Spec} C(X)$.

Let now $\mathfrak{p} \in \operatorname{Spec} C(S)-\mathrm{V}\left(I_{R}\right)$. Take $g \in I_{R}-\mathfrak{p}$. If $U=\operatorname{coz}(g)$, then by 1.1 we have

$$
\begin{aligned}
C(S)_{\mathfrak{p}} & =\left(C(S)_{g}\right)_{\mathfrak{p}}=\left(C^{*}(S)_{g}\right)_{\mathfrak{p}}=\left(C^{*}(U)_{g}\right)_{\mathfrak{p}}, \\
C(X)_{\mathfrak{p}} & =\left(C^{*}(X)_{g}\right)_{\mathfrak{p}}=\left(C^{*}\left(\pi^{-1}(U)\right)_{g}\right)_{\mathfrak{p}}
\end{aligned}
$$

Since $U=\operatorname{coz}(g) \subseteq S_{0}$, the covering $\pi: \pi^{-1}(U) \rightarrow U$ is unbranched and so, by 3.2, the map Spec $C(X)_{\mathfrak{p}} \rightarrow \operatorname{Spec} C(S)_{\mathfrak{p}}$ is a finite covering. Since the fibre over $\mathfrak{p}$ under this map is just $\pi_{s}^{-1}(\mathfrak{p})$, we may conclude that any two points in $\pi_{s}^{-1}(\mathfrak{p})$ have disjoint neighbourhoods in $\operatorname{Spec} C(S)$. 
Finally, we prove that the fibres of $\pi_{s}$ have no more than $n$ points using the arguments given in 3.2 for $\pi_{\beta}$.

REMARK 3.4. It is easy to see that if the extension $\pi_{\beta}: \beta X \rightarrow \beta S$ of an unbranched covering $\pi: X \rightarrow S$ is also an unbranched covering then $\beta S$ has a finite cover by cozero-sets over which $\pi_{\beta}$ is a trivial covering, and consequently $\pi$ is trivial on a finite cover of $S$ by cozero-sets. Conversely, if an unbranched covering $\pi: X \rightarrow S$ is trivial on a finite cover of $S$ by cozero-sets then it is not difficult to prove that $\pi_{\beta}: \beta X \rightarrow \beta S$ and $\pi_{s}$ : Spec $C(X) \rightarrow$ Spec $C(S)$ are also unbranched coverings, since in this case $C(X)$ is a locally trivial $C(S)$-algebra (see the proof of $5.8(\mathrm{ii})$ ).

4. Flatness. Recall that a ring homomorphism $h: A \rightarrow B$ is said to be flat if $B$ with the structure of $A$-module induced by $h$ is flat, i.e., the tensor product $\otimes_{A} B$ is an exact functor.

Proposition 4.1. If $\pi: X \rightarrow S$ is a covering of finite degree then the induced homomorphism $C(S) \rightarrow C(X)$ is flat.

Pr o of (First step). We use induction on $n$, the degree of $\pi$. For $n=1$ the assertion is trivially true. Assume that it is true when the degree is less than $n$.

Recall that the homomorphism $C(S) \rightarrow C(X)$ is flat if and only if for every $\mathfrak{p} \in \operatorname{Spec} C(S)$ and for every $\mathfrak{q} \in \pi_{s}^{-1}(\mathfrak{p})$ the local homomorphism $C(S)_{\mathfrak{p}} \rightarrow C(X)_{\mathfrak{q}}$ is flat [13, Theorem 7.1].

If $\pi_{s}^{-1}(\mathfrak{p})=\left\{\mathfrak{q}_{1}, \ldots, \mathfrak{q}_{m}\right\}$ and $m \geq 2$, we can take a basic neighbourhood $\mathrm{D}(g)$ of $\mathfrak{p}$ special for $\pi_{s}$ such that

$$
\pi_{s}^{-1}(\mathrm{D}(g))=\mathrm{D}\left(g_{1}\right) \sqcup \ldots \sqcup \mathrm{D}\left(g_{m}\right),
$$

where each $\mathrm{D}\left(g_{i}\right)$ is a neighbourhood of $\mathfrak{q}_{i}$ such that $\pi_{s}\left(\mathrm{D}\left(g_{i}\right)\right)=\mathrm{D}(g)$. It is clear that we can take $g$ and $g_{i}$ to be in $C^{*}(S)$ and $C^{*}(X)$, respectively.

If $U=\operatorname{coz}(g)$ and $U_{i}=\operatorname{coz}\left(g_{i}\right)$, then $\pi: U_{i} \rightarrow U$ is a covering of degree less than or equal to $n-(m-1)$ and, by Proposition 3.2, the extension $\pi_{\beta}: \beta U_{i} \rightarrow \beta U$ is also a covering of the same degree. Then, by the induction hypothesis, $C(\beta U)=C^{*}(U) \rightarrow C^{*}\left(U_{i}\right)=C\left(\beta U_{i}\right)$ is flat, and therefore so is $C^{*}(S)_{g}=C^{*}(U)_{g} \rightarrow C^{*}(X)_{g_{i}}=C^{*}\left(U_{i}\right)_{g_{i}}$.

By 3.1(ii), $C(S)_{g}$ and $C(X)_{g_{i}}$ are the rings of fractions of $C^{*}(S)_{g}$ and $C^{*}(X)_{g_{i}}$ respectively, with respect to the multiplicatively closed subset $M_{S}$ $=\left\{f \in C^{*}(S): 0 \notin f(S)\right\}$, and $C(S)_{g} \rightarrow C(X)_{g_{i}}$ is just the homomorphism between the rings of fractions induced by

$$
C^{*}(S)_{g}=C^{*}(U)_{g} \rightarrow C^{*}(X)_{g_{i}}=C^{*}\left(U_{i}\right)_{g_{i}} .
$$

Hence, $C(S)_{g} \rightarrow C(X)_{g_{i}}$ is flat, and consequently so is $C(S)_{\mathfrak{p}} \rightarrow C(X)_{\mathfrak{q}_{i}}$.

Thus, we have proved that, except for those points $\mathfrak{p} \in \operatorname{Spec} C(S)$ which have only one point $\mathfrak{q}$ in their $\pi_{s}$-fibre, the homomorphism $C(S)_{\mathfrak{p}} \rightarrow C(X)_{\mathfrak{q}}$ 
is flat. That is, we have proved that for every ideal $I$ in $C(S)$ the kernel of the natural homomorphism

$$
I \otimes_{C(S)} C(X) \rightarrow C(X),
$$

which is just $N=\operatorname{Tor}_{1}(C(X), C(S) / I)$, is a $C(X)$-module such that $N_{\mathfrak{q}}=0$ for every point $\mathfrak{q} \in \operatorname{Spec} C(X)$ where the index of branching of $\pi_{s}$ is less than $n$. Or, in other words, the support of the $C(X)$-module $N, \operatorname{Supp}(N)=$ $\left\{\mathfrak{p} \in \operatorname{Spec} C(X): N_{\mathfrak{p}} \neq 0\right\}$, is contained in the closed subset of $\operatorname{Spec} C(X)$ consisting of the points where the index of branching of $\pi_{s}$ is $n$. To proceed with the proof, therefore, we need to identify this closed subset.

Definitions 4.2. Let $\pi: X \rightarrow S$ be a covering of degree $n$. The trace of a function $f \in C(X)$ is defined to be the continuous function on $S$

$$
\operatorname{tr}(f)(s)=\frac{1}{n} \sum_{x \in \pi^{-1}(s)} b(x) f(x)
$$

where $b(x)$ denotes the index of branching of $\pi$ at $x$.

The map $\operatorname{tr}: C(X) \rightarrow C(S)$ that sends $f$ to $\operatorname{tr}(f)$ is a homomorphism of $C(S)$-modules that is the identity over $C(S)$. Hence, we have an isomorphism between $C(X)$ and $C(S) \oplus \operatorname{Ker}(\operatorname{tr})$ because we may write each $f \in C(X)$ as $f=(f-\operatorname{tr}(f))+\operatorname{tr}(f)$.

We denote by $K$ the ideal in $C(X)$ generated by $\operatorname{Ker}(\operatorname{tr})$.

Lemma 4.3. Let $\pi: X \rightarrow S$ be a covering of degree $n$, and let $F$ be the closed subset in $\operatorname{Spec} C(X)$ consisting of the points where the index of branching of $\pi_{s}: \operatorname{Spec} C(X) \rightarrow \operatorname{Spec} C(S)$ is $n$.

(i) Every minimal element $\mathfrak{q}$ in $F$ is a z-ideal in $C(X)$.

(ii) $F=V(K)$.

Proof. (i) Let $\mathfrak{q}$ be a minimal element in $F$ and $\mathfrak{p}=\pi_{s}(\mathfrak{q})$. If $\mathfrak{q}$ is a minimal prime ideal in $C(X)$ then it is a z-ideal [6, Theorem 14.7]. If this is not the case, we may take a minimal prime ideal $\mathfrak{n}_{1}$ contained in $\mathfrak{q}$. Since $\pi_{s}$ is open, the prime ideal $\mathfrak{n}=\pi_{s}\left(\mathfrak{n}_{1}\right)$ is minimal in $C(S)$, and clearly $\mathfrak{n} \subseteq \mathfrak{p}$. Let $\mathfrak{n}_{1}, \ldots, \mathfrak{n}_{m}$ be the points of the fibre over $\mathfrak{n}$. They are minimal prime ideals in $C(X)$, because $\pi_{s}$ is separated and so there is no inclusion between prime ideals belonging to the same fibre. Since $\pi_{s}$ is closed, $\pi_{s}\left(\mathrm{~V}\left(\mathfrak{n}_{i}\right)\right)$ contains the closure $\mathrm{V}(\mathfrak{n})$ of $\mathfrak{n}=\pi_{s}\left(\mathfrak{n}_{i}\right)$, i.e., $\pi_{s}\left(\mathrm{~V}\left(\mathfrak{n}_{i}\right)\right)=\mathrm{V}(\mathfrak{n})$, and as $\mathfrak{q}$ is the unique prime ideal in $C(X)$ such that $\pi_{s}(\mathfrak{q})=\mathfrak{p}$, we see that the prime ideals $\mathfrak{n}_{1}, \ldots, \mathfrak{n}_{m}$ are all contained in $\mathfrak{q}$, and consequently $\mathfrak{n}_{1}+\ldots+\mathfrak{n}_{m} \subseteq \mathfrak{q}$.

The ideal $\mathfrak{n}_{1}+\ldots+\mathfrak{n}_{m}$ is prime $[6$, Problem $14 \mathrm{~B}]$ and a z-ideal $[6,14.8$ Lemma]. It is the unique point in the fibre over $\pi_{s}\left(\mathfrak{n}_{1}+\ldots+\mathfrak{n}_{m}\right)$, because if another point $\mathfrak{q}^{\prime} \in \operatorname{Spec} C(X)$ is in the same fibre then, since $\pi_{s}$ is open, $\mathfrak{q}^{\prime}$ contains one of the ideals $\mathfrak{n}_{i}$. But this cannot occur because, on the one hand, the prime ideals containing $\mathfrak{n}_{i}$ form a chain $[6,14.3(\mathrm{c})]$, and on the other 
hand, since $\pi_{s}$ is separated, there is no inclusion between $\mathfrak{q}^{\prime}$ and $\mathfrak{n}_{1}+\ldots+\mathfrak{n}_{m}$. Thus, by the choice of $\mathfrak{q}$, we have $\mathfrak{q}=\mathfrak{n}_{1}+\ldots+\mathfrak{n}_{m}$ and hence $\mathfrak{q}$ is a z-ideal.

(ii) To show that $F \subseteq \mathrm{V}(K)$, it suffices to prove that every minimal element $\mathfrak{q}$ of $F$ belongs to $\mathrm{V}(K)$, i.e., $\mathfrak{q}$ contains every function with null trace. Let $f \in \operatorname{Ker}(\operatorname{tr}), f^{+}=\sup (f, 0)$ and $f^{-}=-\inf (f, 0)$. Since $f^{+} \cdot f^{-}$ $=0$, one of the functions $f^{+}$or $f^{-}$belongs to $\mathfrak{q}$. Denote this function by $g$, and define $g^{*}$ on $S$ by $g^{*}(s)=\sup \left\{g(x): x \in \pi^{-1}(s)\right\} \quad\left(g^{*}\right.$ is clearly continuous). Since $0 \leq g$ one can prove that $\pi_{s}(\mathrm{D}(g))=\mathrm{D}\left(g^{*}\right)$ (see the proof of [14, Corollary 1.6]). As $\mathfrak{q}$ is the unique point in the fibre over $\pi_{s}(\mathfrak{q})$, we see that $g^{*} \in \pi_{s}(\mathfrak{q})$ and hence $g^{*} \in \mathfrak{q}$. Moreover, since $\operatorname{tr}(f)=0$, the sign of $f$ changes in every fibre of $\pi$ so that $\mathrm{Z}\left(g^{*}\right) \subseteq \mathrm{Z}(f)$. As $\mathfrak{q}$ is a z-ideal, this implies that $f \in \mathfrak{q}$.

Conversely, to prove that $\mathrm{V}(K) \subseteq F$, consider a point $\mathfrak{q} \in \mathrm{V}(K)$ and suppose that $\mathfrak{q}^{\prime}$ is another prime ideal in $C(X)$ such that $\pi_{s}(\mathfrak{q})=\pi_{s}\left(\mathfrak{q}^{\prime}\right)$. If $f \in \mathfrak{q}$ then, since $f-\operatorname{tr}(f) \in \mathfrak{q}$, one has

$$
\operatorname{tr}(f) \in \mathfrak{q} \cap C(S)=\pi_{s}(\mathfrak{q})=\pi_{s}\left(\mathfrak{q}^{\prime}\right)
$$

and therefore $\operatorname{tr}(f) \in \mathfrak{q}^{\prime}$. If $f \geq 0$ then $0 \leq f \leq n \cdot \operatorname{tr}(f)$, and by the convexity of $\mathfrak{q}^{\prime}\left[6\right.$, Theorem 5.5], this implies that $f \in \mathfrak{q}^{\prime}$. Thus $\mathfrak{q} \subseteq \mathfrak{q}^{\prime}$, and since $\pi_{s}$ is separated, we conclude that $\mathfrak{q}=\mathfrak{q}^{\prime}$.

Corollary 4.4. If $\pi: X \rightarrow S$ is a covering of finite degree, then $K$, the ideal in $C(X)$ generated by the functions with null trace, is a z-ideal.

Proof. This follows from 4.3 because, as is shown in [14, Corollary 2.5], if every prime ideal minimal in $\mathrm{V}(K)$ is a z-ideal, then so is $K$.

Continuation of the proof of Proposition 4.1. We have proved that for every ideal $I$ in $C(S)$ the $C(X)$-module $N=\operatorname{Tor}_{1}(C(X), C(S) / I)$ has its support contained in the closed subset of Spec $C(X)$ consisting of the points where the index of branching of $\pi_{s}$ is $n$, i.e., $\operatorname{Supp}(N) \subseteq \mathrm{V}(K)$. Since $K$ is a z-ideal, this implies that $K \cdot N=0$ (see [14, Proposition 2.7]).

If $\sum g_{i} \otimes f_{i}$ is an element of $N$ then, by the definition of $N$, one has $\sum g_{i} \cdot f_{i}=0$ and therefore $\sum g_{i} \cdot \operatorname{tr}\left(f_{i}\right)=0$. Thus, we can write $\sum g_{i} \otimes f_{i}=$ $\sum g_{i} \otimes\left(f_{i}-\operatorname{tr}\left(f_{i}\right)\right)$ and replacing $f_{i}$ by $f_{i}-\operatorname{tr}\left(f_{i}\right)$ we can take $f_{i}$ such that $\operatorname{tr}\left(f_{i}\right)=0$, i.e., $f_{i} \in K$. It is proved in [14] that, since $K$ is a z-ideal, there exist functions $h, f_{i}^{\prime} \in K$ such that

$$
\mathrm{Z}(h)=\mathrm{Z}\left(f_{1}\right) \cap \ldots \cap \mathrm{Z}\left(f_{n}\right), \quad \mathrm{Z}\left(f_{i}^{\prime}\right)=\mathrm{Z}\left(f_{i}\right), \quad f_{i}=h^{2} \cdot f_{i}^{\prime} .
$$

Since $\sum g_{i} \cdot h \cdot f_{i}^{\prime}=0$, we see that $\sum g_{i} \otimes h \cdot f_{i}^{\prime} \in N$, and hence

$$
\sum g_{i} \otimes f_{i}=h \cdot \sum g_{i} \otimes h \cdot f_{i}^{\prime} \in K \cdot N .
$$

Thus we have proved that $N=K \cdot N=0$, and, by the ideal criterion [13, Theorem 7.7], that $C(X)$ is a flat $C(S)$-module. 


\section{Characterization of finite coverings}

Theorem 5.1. If $\pi: X \rightarrow S$ is a covering of finite degree, then the induced homomorphism $C(S) \rightarrow C(X)$ is integral and flat.

Pr o of. This follows from Propositions 2.8 and 4.1.

REMARK 5.2. If a finite covering $\pi: X \rightarrow S=X / G$ is the natural projection map of a topological space $X$ onto the quotient space by an action of a finite group $G$, then $G$ acts in a natural way on the ring $C(X)$, and consequently on the topological space Spec $C(X)$. It is not difficult to see that the subring of $G$-invariant elements is just $C(X)^{G}=C(S)$ and that $\operatorname{Spec} C(X) / G=\operatorname{Spec} C(X)^{G}=\operatorname{Spec} C(S)$. Hence, the induced homomorphism $C(X) \rightarrow C(S)$ is trivially integral and $\pi_{s}: \operatorname{Spec} C(X) \rightarrow \operatorname{Spec} C(S)$ is just the natural projection map, so that it is also a finite covering. Moreover, one may repeat the arguments given in Proposition 4.1 and prove that $C(S) \rightarrow C(X)$ is flat. Thus, Theorem 5.1 also holds for such coverings $\pi: X \rightarrow S=X / G$.

Now, our aim is to prove the converse of 5.1. In the following propositions we start from algebraic properties of the homomorphism $C(S) \rightarrow C(X)$ to obtain topological properties for the corresponding map $X \rightarrow S$.

Proposition 5.3. Let $\pi: X \rightarrow S$ be a map between locally compact metric spaces. If the induced homomorphism $C(S) \rightarrow C(X)$ is flat, then $\pi$ is open.

Proof. If $C(S) \rightarrow C(X)$ is flat then, for every $x \in X$, the local homomorphism $C(S)_{s} \rightarrow C(X)_{x}$ (where $s=\pi(x)$ ) is also flat. Since $\mathfrak{m}_{s} \cdot C(X) \subseteq$ $\mathfrak{m}_{x}$, this homomorphism is faithfully flat, and therefore injective. Then, as is proved in [7], $\pi$ carries every neighbourhood of $x$ onto a neighbourhood of $s$.

Proposition 5.4. Let $\pi: X \rightarrow S$ be a map between realcompact spaces. If the induced homomorphism $C(S) \rightarrow C(X)$ is integral then $\pi$ is finite and $\pi_{s}: \operatorname{Spec} C(X) \rightarrow \operatorname{Spec} C(S)$ is proper and separated.

Proof. We first prove that $\pi$ is proper, or equivalently, that $\pi_{\beta}: \beta X \rightarrow$ $\beta S$ transforms $\beta X-X$ into $\beta S-S$ [5, Theorem 3.7.16]. Since $X$ is realcompact, a point in $\beta X$ belongs to $X$ if and only if the corresponding maximal ideal $\mathfrak{m}$ is real, i.e., $C(X) / \mathfrak{m}=\mathbb{R}$. If $\mathfrak{m}^{\prime}=\pi_{\beta}(\mathfrak{m})$ then $C(S) / \mathfrak{m}^{\prime} \rightarrow C(X) / \mathfrak{m}$ is an algebraic extension, because $C(S) \rightarrow C(X)$ is integral. Since every residue field of a ring of continuous functions is totally ordered [6, Theorem 5.5] and real-closed [6, Theorem 13.4], we have $C(X) / \mathfrak{m}=\mathbb{R}$, or equivalently $\mathfrak{m}=\mathfrak{m}_{x}$ for some $x \in X$, if and only if $C(S) / \mathfrak{m}^{\prime}=\mathbb{R}$, i.e., $\mathfrak{m}^{\prime}=\mathfrak{m}_{s}$ for some $s \in S$. This proves that $\pi_{\beta}(\beta X-X)=\beta S-S$. 
Now, as $\pi$ is proper, every fibre $\pi^{-1}(s)$ is a compact subspace of $X$. Thus, every continuous function on $\pi^{-1}(s)$ can be extended to the whole $X[6$, 3.11.(c)]. In other words, the restriction homomorphism $C(X) \rightarrow C\left(\pi^{-1}(s)\right)$ is surjective. This implies that the homomorphism $C(\{s\})=\mathbb{R} \rightarrow C\left(\pi^{-1}(s)\right)$ is also integral, i.e., every function in $C\left(\pi^{-1}(s)\right)$ is a root of a polynomial with coefficients in $C(\{s\})=\mathbb{R}$, and therefore such functions have finite range. Hence, the set $\pi^{-1}(s)$ is finite [6, Problem 3L]. Thus, $\pi$ is finite.

The fibres of the map between the prime spectra induced by a ring homomorphism are always compact (not necessarily Hausdorff). If the ring homomorphism is integral then the corresponding map is closed [1, Chapter 5, Exercise 1] and there is no inclusion between prime ideals belonging to the same fibre [1, Corollary 5.9]. This latter property implies (see the proof of 3.2) that if $C(S) \rightarrow C(X)$ is integral, then $\pi_{s}: \operatorname{Spec} C(X) \rightarrow \operatorname{Spec} C(S)$ is separated.

REMARK 5.5. It is not difficult to prove that if $\pi: X \rightarrow S$ is a finite covering and the induced homomorphism $C(S) \rightarrow C(X)$ is integral, then the supremum of the cardinalities of the fibres of $\pi$ is finite. Explicitly: suppose that, on the contrary, for every $n \in \mathbb{N}$ there are points in $S$ with $n$ or more points in their fibres. Then $S$ contains a countably infinite discrete subset $S^{\prime}=\left\{s_{n}\right\}$ such that $\pi^{-1}\left(s_{n}\right)$ has $n$ or more points. Consider in $X$ the countably infinite discrete subset $X^{\prime}=\pi^{-1}\left(S^{\prime}\right)=\left\{x_{m}\right\}$. If $\left\{r_{m}\right\}$ is a convergent sequence of real numbers such that $r_{m} \neq r_{k}$ whenever $m \neq k$, then there exists a continuous function $f$ on $X$ such that $f\left(x_{m}\right)=r_{m}[6$, Problem 3L]. It is clear that $f$ cannot be a root of any polynomial with coefficients in $C(S)$. This contradicts the hypothesis that $C(X)$ is integral over $C(S)$.

Recall that a ring homomorphism $h: A \rightarrow B$ is said to be finite if $B$ with the structure of $A$-module induced by $h$ is finitely generated.

Proposition 5.6. Let $\pi: X \rightarrow S$ be a map between realcompact spaces. If the induced homomorphism $C(S) \rightarrow C(X)$ is finite, then $\pi: X \rightarrow S$, $\pi_{\beta}: \beta X \rightarrow \beta S$ and $\pi_{s}: \operatorname{Spec} C(X) \rightarrow \operatorname{Spec} C(S)$ are finite and locally injective.

Proof. Every finite ring homomorphism $h: A \rightarrow B$ has the following properties (see [1]): the fibres of the induced continuous map $h^{*}: \operatorname{Spec} B \rightarrow$ Spec $A$ are finite; if $\mathfrak{m}$ is a maximal ideal in $B$ then $h^{*}(\mathfrak{m})$ is a maximal ideal in $A$ and $\mathfrak{m} \cdot B_{\mathfrak{m}}$ is a unique prime ideal in $B_{\mathfrak{m}}$ that contains $h^{*}(\mathfrak{m}) \cdot B_{\mathfrak{m}}$.

Thus, if the induced homomorphism $C(S) \rightarrow C(X)$ is finite, then the fibres of $\pi_{s}$ are finite and, by 5.4 , this map is separated, so that $\pi_{s}$ is finite and then so are $\pi$ and $\pi_{\beta}$. 
To see that these maps are locally injective, it suffices to prove that every point $\mathfrak{m}$ in the maximal spectrum of $C(X)$ has a neighbourhood in Spec $C(X)$ where $\pi_{s}$ is injective, since every prime ideal $\mathfrak{p}$ is contained in some maximal ideal $\mathfrak{m}$ and then every neighbourhood of $\mathfrak{m}$ is also a neighbourhood of $\mathfrak{p}$.

If $\mathfrak{m}^{\prime}=\pi_{s}(\mathfrak{m})$ then $\mathfrak{m}^{\prime}$ is a maximal ideal in $C(S)$ and the homomorphism between the residue class fields $C(S) / \mathfrak{m}^{\prime} \rightarrow C(X) / \mathfrak{m}$ is also finite. Moreover, since $\mathfrak{m} \cdot C(X)_{\mathfrak{m}}$ is the unique prime ideal in $C(X)_{\mathfrak{m}}$ that contains $\mathfrak{m}^{\prime} \cdot C(X)_{\mathfrak{m}}$ and $\mathfrak{m}$ is a z-ideal, it can be proved that $\mathfrak{m}^{\prime} \cdot C(X)_{\mathfrak{m}}=\mathfrak{m} \cdot C(X)_{\mathfrak{m}}$ [14, Corollary 2.6]. Thus, the quotient ring $C(X)_{\mathfrak{m}} / \mathfrak{m}^{\prime} \cdot C(X)_{\mathfrak{m}}$ is just the field $C(X)_{\mathfrak{m}} / \mathfrak{m} \cdot C(X)_{\mathfrak{m}}=C(X) / \mathfrak{m}$. This field $C(X) / \mathfrak{m}$ is totally ordered [6, Theorem 5.5] and $C(S) / \mathfrak{m}^{\prime}$ is real-closed [6, Theorem 13.4], so that the degree of the field extension $C(S) / \mathfrak{m}^{\prime} \rightarrow C(X) / \mathfrak{m}$ is one. Since the homomorphism between the local rings $C(S)_{\mathfrak{m}^{\prime}} \rightarrow C(X)_{\mathfrak{m}}$ is finite, we can apply Nakayama's Lemma [13, Proposition 2.8] to conclude that this homomorphism is surjective. It is not difficult to prove that, since $C(S) \rightarrow C(X)$ is finite, there exist $g \in C(S)-\mathfrak{m}^{\prime}$ and $f \in C(X)-\mathfrak{m}$ such that the induced homomorphism between the rings of fractions $C(S)_{g} \rightarrow C(X)_{g \cdot f}$ is surjective, and consequently $\operatorname{Spec} C(X)_{g \cdot f} \rightarrow \operatorname{Spec} C(S)_{f}$ is injective. Since Spec $C(X)_{g \cdot f}=\mathrm{D}(g \cdot f)$ is a neighbourhood of $\mathfrak{m}$, this proves that $\pi_{s}$ is locally injective.

REMARK 5.7. It follows from 5.6 that the obstruction to the finiteness of the homomorphism $C(S) \rightarrow C(X)$ induced by a finite covering $\pi: X \rightarrow S$ is just the existence of the branched set. Moreover, for a branching point $x$, even the local homomorphism $C(S)_{s} \rightarrow C(X)_{x}$ (where $s=\pi(x)$ ) is not finite. If this local homomorphism is finite, then with the same arguments given in 5.6, one can prove that it is surjective. And, as is shown in [7], for locally compact metric spaces $X$ and $S$, this implies that $\pi$ is injective in some neighbourhood of $x$.

TheOrem 5.8. Let $X$ and $S$ be topological manifolds (Hausdorff and paracompact).

(i) A continuous map $\pi: X \rightarrow S$ is a finite covering if and only if the induced homomorphism $C(S) \rightarrow C(X)$ is integral and flat.

(ii) A continuous map $\pi: X \rightarrow S$ is an unbranched finite covering if and only if the induced homomorphism $C(S) \rightarrow C(X)$ is finite and flat.

Proof. (i) As was noted in 2.7, every finite covering between manifolds is a covering of finite degree. Thus, the necessity follows from Theorem 5.1, and the sufficiency from Propositions 5.4 and 5.3.

(ii) It is not difficult to prove that, since $S$ has finite (cover) dimension, every open cover of $S$ has a refinement that may be written as the union of 
finitely many families consisting of pairwise disjoint open sets. Hence, $S$ has a finite cover by open sets $U$ such that $\pi: \pi^{-1}(U) \rightarrow U$ is a trivial covering. Every open subset of $S$ is a cozero-set $U=\operatorname{coz}(g)$. If $\pi^{-1}(U)=U_{1} \sqcup \ldots \sqcup U_{n}$, where each $U_{i}$ is homeomorphic to $U$ under $\pi$, then the functions $g_{i}$ defined by $g_{i}=g$ on $U_{i}$ and $g_{i}=0$ on $X-U_{i}$ are continuous on $X$. Since $g_{i} \cdot g_{j}=0$ if $i \neq j, g^{2}=\sum g_{i}^{2}$, and every prime ideal in $C(X)$ is convex [6, Theorem $5.5]$, one has the following decomposition of $\operatorname{Spec} C(X)_{g}$ :

$$
\text { Spec } C(X)_{g}=\mathrm{D}(g)=\mathrm{D}\left(g_{1}\right) \sqcup \ldots \sqcup \mathrm{D}\left(g_{n}\right) .
$$

Hence, $C(X)_{g}=C(X)_{g_{1}} \times \ldots \times C(X)_{g_{n}}[3$, Chap. II, $\S 2$, no. 3, Proposition $15]$.

The natural homomorphism $C(S)_{g} \rightarrow C(X)_{g_{i}}$ is an isomorphism, because it is a localization of $C^{*}(S)_{g}=C^{*}(U)_{g} \rightarrow C^{*}(X)_{g_{i}}=C^{*}\left(U_{i}\right)_{g_{i}}$ (see $3.1(\mathrm{ii}))$ and the latter is clearly an isomorphism. Thus, $C(X)_{g}$ is a trivial $C(S)_{g}$-algebra, i.e., $C(X)_{g}=C(S)_{g} \times \ldots \times C(S)_{g}$. Since $S$ is covered by a finite number of these cozero-sets $U$, we conclude that $C(X)$ is a finite, and of course flat, $C(S)$-algebra.

Conversely, if $C(S) \rightarrow C(X)$ is finite and flat, then $\pi: X \rightarrow S$ is a finite covering, as we have proved in (i), and it is unbranched by 5.6.

Acknowledgments. This paper is based in part on the author's doctoral dissertation, written under the supervision of Professor J. B. Sancho de Salas. The author wishes to express her gratitude to Professors J. A. Navarro González and J. B. Sancho de Salas for the valuable advice and encouragement given during the preparation of this paper.

\section{References}

[1] M. Atiyah and I. G. MacDonald, Introduction to Commutative Algebra, Addison-Wesley, 1969.

[2] R. L. Blair and A. W. Hagger, Extensions of zero-sets and of real-valued functions, Math. Z. 136 (1974), 41-52.

[3] N. Bourbaki, Algèbre Commutative, Chs. 1 and 2, Hermann, 1961.

[4] V. I. Danilov, Algebraic varieties and schemes, in: Algebraic Geometry I, I. R. Shafarevich (ed.), Encyclopaedia Math. Sci. 23, Springer, 1994.

[5] R. Engelking, General Topology, Heldermann, 1989.

[6] L. Gillman and M. Jerison, Rings of Continuous Functions, Springer, 1976.

[7] K. R. Good earl, Local isomorphisms of algebras of continuous functions, J. London Math. Soc. (2) 16 (1977), 348-356.

[8] A. Grothendieck, Éléments de Géométrie Algébrique IV, Inst. Hautes Études Sci. Publ. Math. 28 (1966).

[9] T. Isiwata, Mappings and spaces, Pacific J. Math. 20 (1967), 455-480.

[10] L. F. Mc Auley and E. E. Robinson, Discrete open and closed maps on generalized continua and Newman's property, Canad. J. Math. 36 (1984), 1081-1112.

[11] B. Malgrange, Ideals of Differentiable Functions, Oxford Univ. Press, 1966. 
[12] W. S. Massey, Algebraic Topology: An Introduction, Springer, 1967.

[13] H. Matsumura, Commutative Ring Theory, Cambridge Univ. Press, 1986

[14] M. A. Mulero, Algebraic properties of rings of continuous functions, Fund. Math. 149 (1996), 55-66.

[15] - Rings of continuous functions and the branch set of a covering, Proc. Amer. Math. Soc. 126 (1998), 2183-2189.

[16] J. C. Tougeron, Idéaux de Fonctions Différentiables, Springer, 1972.

Departamento de Matemáticas

Universidad de Extremadura

06071 Badajoz, Spain

E-mail: mamulero@unex.es

Received 19 August 1997;

in revised form 22 June 1998 